\title{
Inertial Force on Floating Inclusion Particles at the Interface of Liquid Steel and Inert Gas
}

\author{
Zilong QIU, ${ }^{*}$ Annelies MALFLIET, Muxing GUO and Bart BLANPAIN \\ Department of Materials Engineering, KU Leuven, Kasteelpark Arenberg 44 box 2450, Leuven, 3001 Belgium. \\ (Received on November 26, 2020; accepted on April 7, 2021; J-STAGE Advance published date: June \\ 18, 2021)
}

\begin{abstract}
To predict the clustering of inclusions at the liquid steel and inert gas interface, it is essential to calculate the pairwise attractive (inertial) force on the inclusions correctly. In most of the studies, the inertial force is calculated from three successive inter-particle distances with a certain time interval, in which the inertial force is assumed to be identical at the first two distances. Considering the nature of the attractive force, a new iteration scheme is proposed where the inertial force is assumed to be non-identical from point to point. However, both the identical and non-identical force schemes tend to give an unreasonably oscillating force when the measured inter-particle distance is less accurate. Moreover, the curve fitting function is also considered in this study including the use of polynomial, exponential, sum of sines, rational, Gaussian functions and Fourier series. Among these functions, the 4th order polynomial and the 2nd order sine are the most robust functions in predicting the inertial force on particles, even when the measured distance is less accurate.
\end{abstract}

KEY WORDS: capillary force; inertial force; clustering; confocal scanning laser microscopy; curve fitting.

\section{Introduction}

Steel cleanliness improvement by reducing non-metallic inclusions (NMI) is an important aspect of high-quality steel production. The NMI in a steel melt are generated either during the deoxidation process or during reoxidation with the environment (atmosphere, slag and refractory). ${ }^{1)}$ Complete removal of these inclusion particles is time-consuming and difficult in practice. Inert gas injection is one of the most effective ways to remove the inclusions by enhancing bubble adherence and agitating turbulence flow to improve inclusion collision and flotation. ${ }^{2-4)}$ Clusters formed by the agglomeration of small inclusions are more readily floated to the melt surface or steel/slag interface. However, it is more detrimental if these clusters remain in the solidified steel. ${ }^{5)}$ During the inert gas injection, the interface of liquid steel and inert gas is an important site for inclusion clustering, where the deformation of the interface is usually detected inducing a strong lateral attractive force between the inclusions. ${ }^{67)}$ Thus, a better understanding of the clustering behavior at the liquid steel and inert gas interface is essential for steel cleanliness control. For this purpose, many in-situ real-time observations of inclusion clustering on steel melt surface were performed with the help of high-temperature confocal scanning laser microscopy

\footnotetext{
* Corresponding author: E-mail: zilg.qiu@gmail.com
}

(CSLM). ${ }^{6,8-13)}$ The pioneering experiment was done by Yin and co-workers, who studied the collision, agglomeration and clustering of alumina inclusion particles at the liquid steel and argon gas interface. ${ }^{6}$ ) Thereafter, the interaction of more complex types of inclusions was studied using a similar methodology, e.g., inclusions in the $\mathrm{CaO}-\mathrm{Al}_{2} \mathrm{O}_{3}-$ $\mathrm{SiO}_{2},{ }^{6,14)} \mathrm{CaO}-\mathrm{Al}_{2} \mathrm{O}_{3}-\mathrm{MgO}-\mathrm{SiO}_{2}{ }^{11,15)}$ and $\mathrm{CaO}-\mathrm{Al}_{2} \mathrm{O}_{3}-$ $\mathrm{MnO}-\mathrm{SiO}_{2}$ systems. ${ }^{16)}$

The long-range lateral attractive force between inclusion particles is believed to be a kind of capillary force, which arises from the interface deformation due to the presence of particles. ${ }^{6,8)}$ The capillary force can be theoretically calculated either from the interfacial force integration along the three-phase contact line and the pressure over particle surface or from the derivative of the free energy change with respect to the interparticle distance. ${ }^{7,17,18)}$ In the metallurgical field, the lateral capillary force is usually assumed to be balanced by the inertial force calculated from three successive inter-particle distances with a known interval time and the effective particle mass. ${ }^{6,10,14,19)}$ However, this method is very sensitive to the accuracy of the measured inter-particle distance, and therefore the inter-particle distance should be measured very accurately to avoid an unreasonable force oscillation. ${ }^{14,19)}$ To tackle this problem, a curve-fitting approach was proposed to represent the relationship between the inter-particle distance and time by a function. The inertial force on particles is calculated by 
the second derivative of the fitting function with respect to time, and then multiply the result with particles' effective mass. ${ }^{16,20)}$ Wikström et al. used a 2 nd order polynomial to fit the discrete inter-particle distance points. ${ }^{20)}$ As a result, a constant inertial force was derived, which indicates that the inertial force on particles is independent of the inter-particle distance. Whereas, Vantilt et al. applied an exponential fitting function, ${ }^{16)}$ indicating that the inertial force is linearly dependent on the inter-particle distance. Except for the polynomial and exponential functions, other commonly used functions can also be applied, e.g., sum of sines, rational, Gaussian functions and Fourier series. However, the inertial forces predicted from these functions differ from each other significantly. Thus, this paper aims to find a robust and accurate approach to calculate the inertial force on inclusion particles from experimental CSLM image sequence.

\section{Inter-particle Distance}

Prior to the inertial force calculation, the inter-particle distance should be measured. This can be done through a sequence of CSLM images. In the CSLM experiment, the observed view is scanned by a laser beam point by point. The reflected laser, which goes through a pinhole, will be projected on a CRT-monitor and recorded on a videotape/ recorder at a certain rate. For CSLM LM21H-SVF17SP, the maximum recording rate of images can be 30 frames/second with $640 \times 480$ pixels in each image. The dimension of the pixels varies with the screen magnification, e.g., the dimension of the pixels is 0.70 and $0.35 \mu \mathrm{m}$ for a magnification of respectively 700 and 1400 .

So far, the definition of inter-particle distance is not universally agreed upon. Some researchers denoted the distance between two-particle surfaces as the inter-particle distance. ${ }^{6,8,10,11,13,14,16,21)}$ Here, we define it as the inter-surface distance. Other researchers treated the distance between the particle's mass centers as the inter-particle distance. ${ }^{12)}$ This we define as the inter-center distance. Clearly, different definitions give different inter-particle distances.

For the inter-surface distance, many pixels on the particle surface can be chosen as the reference points for the measurement, e.g., pixel pair of $\mathrm{A}$ and $\mathrm{B}$ or pixel pair of $\mathrm{C}$ and D in Fig. 1. The key point of this measurement is that the reference pixels should be tracked throughout the image sequence. Coletti and Vantilt et al. proposed the shortest distance as the inter-surface distance, ${ }^{14,16)}$ in which multitime measurements might be needed to find the pixel pair with the shortest distance. Given the 3D nature of inclusion particles and the opaque property of liquid steel, the recorded inclusion particle in the image is merely the $2 \mathrm{D}$ area which is enclosed by the three-phase contact line. This area highly depends on the immersion depth of the inclusion in liquid steel. Since the deformation of steel surface caused by each inclusion particle tends to reinforce each other when the particles approach each other, this reinforcement results in a three-phase contact line change, i.e., immersion depth change. Therefore, an additional inter-surface distance change may be created by the particle immersion depth change. Moreover, during the clustering, inclusion particles move not only by translation but also by rotation. The torque-induced particle rotation will also contribute to the inter-surface distance change. We can conclude that the individual particle movement of immersion depth change and rotation makes the inter-surface distance method less reliable.

The inter-center distance between inclusions was proposed by Nakajima et al., ${ }^{12)}$ and it was defined as the distance between mass centers of the projected 2D particles. The procedure of finding the particle mass center is shown in Fig. 2. Firstly, the monochrome gray image is converted into a binary image. The threshold of the gray level for binarization is non-trivial in this case. Since the difference of the gray levels between particles and their surrounding background is not always obvious, the standard gray threshold method, such as Otsu's method, ${ }^{22)}$ usually fails to extract the

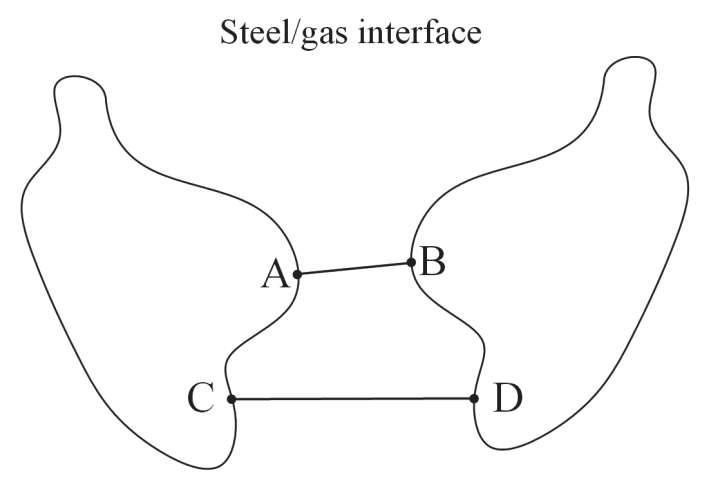

Fig. 1. Schematic of two inclusion particles projected on the steel/ gas interface.

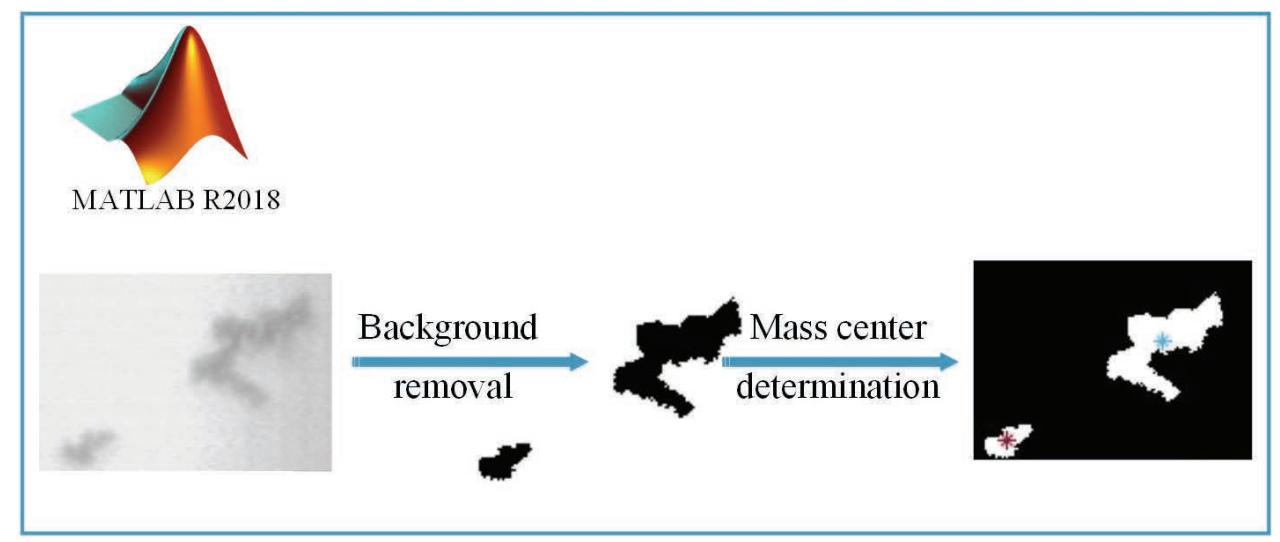

Fig. 2. Procedures of the mass center determination from a 2D CSLM image. (Online version in color.) 
inclusions from the background. Thus, the threshold of gray level needs to be manually adjusted until the background around the target particles is properly removed. Secondly, the mass centers are calculated from the extracted inclusion particles. For the sake of simplicity, the particles are simplified as plates of uniform thicknesses, and the corresponding mass centers are merely 2D centers, which are not necessarily identical with the real $3 \mathrm{D}$ particles. Assuming that a 2D particle in the Cartesian coordinate system with a mass center of $(X \mathrm{c}, Y \mathrm{c})$, the $X \mathrm{c}$ and the $Y \mathrm{c}$ can be calculated by averaging the net $X$ and $Y$ components of all the pixels within the projected particle. Finally, the mass centers of the particles are marked with a 'star', as shown in Fig. 2. This procedure can be easily automated in a MATLAB script.

As mentioned in the previous paragraph, the immersion depth change and the rotation of particles may induce a deviation from the real inter-particle distance. If the three-phase contact line retreats/advances evenly around particles and the rotation is around a vertical axis that goes through the particle mass center, the deviation from the real inter-particle distance will be reduced by adopting the inter-center distance. It is worth noting that the over- and under-estimation of the inter-particle distance are inevitable because of the immersion depth change, particle rotation, image quality etc. This distance deviation will create a deviation in particle velocity calculation and will finally affect the calculated inertial force on particles. In the following calculations, the inter-center distance will be applied as the inter-particle distance.

\section{Inertial Force Calculation Approaches}

Clustering of inclusions at a bubble surface is largely controlled by a long-range lateral attractive force, where the lateral force is confined in the plane of the interface between the gas phase and liquid steel. This attractive force between inclusions is mainly from the capillary interaction induced by the deformation of the meniscus around particles. ${ }^{6,8)}$ If the drag resistance from liquid steel is ignored, this capillary force will be balanced by a virtual inertial force in a non-inertial frame of reference. Thus, accurately calculating the inertial force from the CSLM image sequence is vital to be able to predict the clustering behaviour of inclusion particles.

In the previous study, the inertial force on inclusion particles is mainly derived from three successive inter-particle distances or from curve fittings. ${ }^{6,10,16,20)}$ In the former approach, the inertial force on particles is usually assumed to be identical at the first two successive distances $\left(L_{1}\right.$ and $L_{2}$ ), as shown in Fig. 3. We call this scheme the identical forces scheme. Here, a new approach is proposed that the forces on particles are different at different distances $\left(L_{1}\right.$ and $L_{2}$ ). We call this scheme the non-identical force scheme, see Fig. 4. In the curve-fitting approach, the relationship between the inter-particle distance and time is fitted by a curve function. The particle acceleration is calculated by the second derivative of the function with respect to time.

\subsection{Identical Force Scheme}

In the identical force scheme, two different cases are considered: the position of one particle is fixed, while the other one is free to move, see Fig. 3(a); or both of the particles are free to move, see Fig. 3(b). The mass of the particles are $m_{1}$ and $m_{2}$ with an initial velocity of $v_{1}$ and $v_{2}$; the time interval is $\Delta t$. In the former case, the inertial force on the

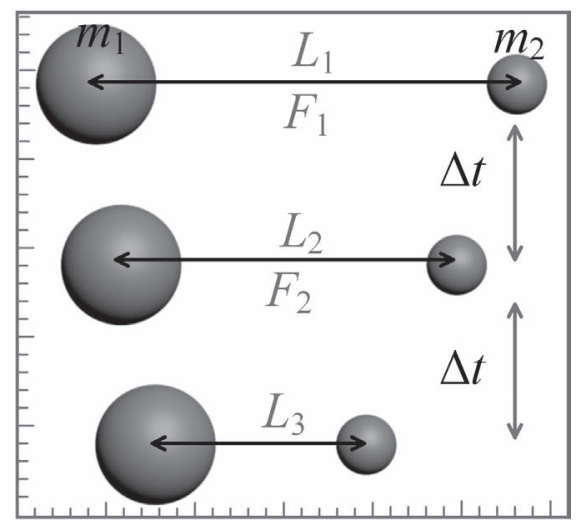

Fig. 4. Schematic of two particles approaching each other under an attractive force. The mass of particle 1 and particle 2 are denoted as $m_{1}$ and $m_{2}$, the initial velocities are $v_{1}$ and $v_{2}$, and the time interval is $\Delta t$. The forces applied on particles during the two-time steps are different as $F_{1}$ and $F_{2}$. a)

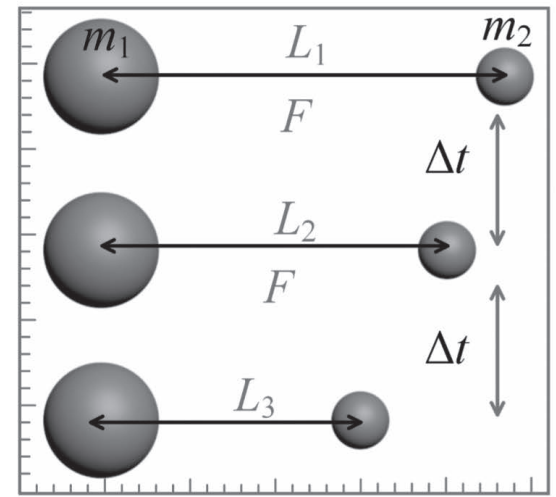

b)

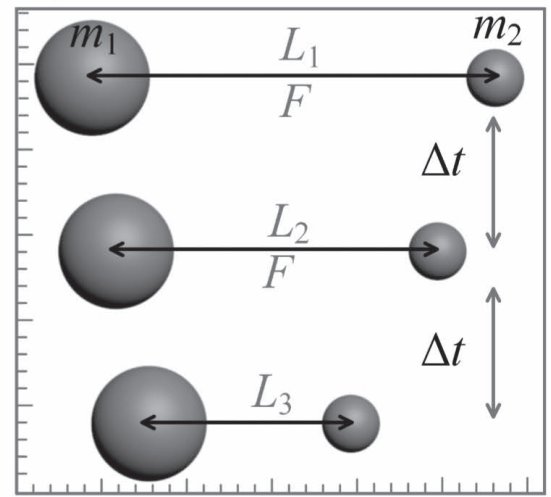

Fig. 3. Schematic of two particles approaching each other under an attractive force. The mass of particle 1 and particle 2 are denoted as $m_{1}$ and $m_{2}$, the initial velocities are $v_{1}$ and $v_{2}$, and the time interval is $\Delta t$. The forces $F$ applied on the particles during the two time steps are identical. a) particle 1 is fixed, particle 2 is free to move; b) particle 1 and particle 2 are free to move. 
moving particles can be calculated through Eqs. (1) to (3). According to Newton's third law of motion, an equivalent force should be felt by the fixed particle. The immobility of a particle may be due to an impediment, such as partially solidified steel or attachment to a crucible wall.

$$
\begin{gathered}
v_{2} \Delta t+\frac{1}{2} \frac{F}{m_{2}} \Delta t^{2}=L_{1}-L_{2}, \ldots \ldots \ldots \\
\left(v_{2}+\frac{F}{m_{2}} \Delta t\right) \Delta t+\frac{1}{2} \frac{F}{m_{2}} \Delta t^{2}=L_{2}-L_{3},
\end{gathered}
$$

By subtracting Eq. (1) from Eq. (2), the inertial force is obtained from Eq. (3),

$$
F=m_{2} \frac{2 L_{2}-L_{1}-L_{3}}{\Delta t^{2}}, \cdots
$$

where $m_{2}$ is the so-called effective mass in this case.

In most situations, without the impediment from the partially solidified steel or crucible wall, two particles should mutually move to each other. The inertial force on the particles can be calculated through Eqs. (4) to (6). It is interesting to note that the difference between Eqs. (3) and (6) is merely dependent on the particle mass by a factor of $m_{1} /\left(m_{1}+m_{2}\right)$.

$$
\begin{gathered}
v_{1} \Delta t+\frac{1}{2} \frac{F}{m_{1}} \Delta t^{2}+v_{2} \Delta t+\frac{1}{2} \frac{F}{m_{2}} \Delta t^{2}=L_{1}-L_{2}, \\
\left(v_{1}+\frac{F}{m_{1}} \Delta t\right) \Delta t+\frac{1}{2} \frac{F}{m_{1}} \Delta t^{2}+ \\
\left(v_{2}+\frac{F}{m_{2}} \Delta t\right) \Delta t+\frac{1}{2} \frac{F}{m_{2}} \Delta t^{2}=L_{2}-L_{3},
\end{gathered}
$$

By subtracting Eq. (4) from Eq. (5), the inertial force is rearranged to be

$$
F=\frac{m_{1} m_{2}}{m_{1}+m_{2}} \frac{2 L_{2}-L_{1}-L_{3}}{\Delta t^{2}},
$$

where $m_{1} m_{2} /\left(m_{1}+m_{2}\right)$ is the so-called effective mass in this case.

\subsection{Non-identical Force Scheme}

According to the capillary theory, the capillary force is proportional to the meniscus slope around particles, while the slope is inversely proportional to the distance between the particle mass centers. ${ }^{7,17)}$ Therefore, the identical force scheme is not theoretically valid and a more accurate iteration scheme is proposed, as shown in Fig. 4. On can see that the attractive force is different at different distances, and we call this scheme the non-identical force scheme. If the two particles are free to move, the non-identical inertial force $F_{2}$ can be calculated from Eqs. (7) to (9). For the case of one fixed particle, the inertial force can be calculated from Eq. (9) by dropping the factor of $m_{1} /\left(m_{1}+m_{2}\right)$. As demonstrated in Eq. (9), the non-identical force scheme needs not only three successive inter-particle distances but also the force $F_{1}$ at the previous distance. Thus, to start the iteration, the nonidentical force scheme is taken to be equal to the identical force scheme. Ideally, this is done for a distance where the slope of the capillary force is still sufficiently small.

$$
\begin{gathered}
v_{1} \Delta t+\frac{1}{2} \frac{F_{1}}{m_{1}} \Delta t^{2}+v_{2} \Delta t+\frac{1}{2} \frac{F_{1}}{m_{2}} \Delta t^{2}=L_{1}-L_{2}, \\
\left(v_{1}+\frac{F_{1}}{m_{1}} \Delta t\right) \Delta t+\frac{1}{2} \frac{F_{2}}{m_{1}} \Delta t^{2}+ \\
\left(v_{2}+\frac{F_{1}}{m_{2}} \Delta t\right) \Delta t+\frac{1}{2} \frac{F_{2}}{m_{2}} \Delta t^{2}=L_{2}-L_{3},
\end{gathered}
$$

By subtracting Eq. (7) from Eq. (8), the inertial force on particles is given

$$
F_{2}=2 \frac{m_{1} m_{2}}{m_{1}+m_{2}} \frac{2 L_{2}-L_{1}-L_{3}}{\Delta t^{2}}-F_{1} .
$$

\subsection{Curve-fitting Method}

Besides the identical and non-identical force schemes, the curve-fitting method was also applied to the inertial force calculation within a specific distance range. ${ }^{16,20)}$ In the curve-fitting method, the relationship between the interparticle distance and time is fitted by a curve function. The acceleration of the particles at different distances is then calculated by a second derivative of the curve function with respect to time. Exponential and 2nd order polynomial functions have been applied by Vantilt et al. and Wikström et al., ${ }^{16,20)}$ and both functions are suitable for fitting the discrete distance points. However, the second derivative of the two functions gives a significantly different result. Therefore, choosing a proper curve function is essential for the curve-fitting method. The commonly used curve functions are polynomials, sum of sines, exponential, rational, Gaussian functions and Fourier series. The performance of these functions on predicting the inertial force should be evaluated further. The different orders of these functions are given from Eqs. (10) to (15). Since we are only interested in the inertia force on particles within a specific range of distance, i.e., from contact to the effective start of the capillary force, the force extracted from the Eqs. (10) to (15) could possibly lead to the real inertial force. It is worth noting that Eqs. (13) and (14) are not the standard exponential and Gaussian functions. Here, the summation form of the exponential and Gaussian functions is used to extend the applicability of these functions to a wider range of situations. We still denote the two equations as the exponential and the Gaussian functions but with different orders.

$$
\begin{aligned}
& L_{\text {Polynomial }}=a_{0}+a_{1} t^{1}+a_{2} t^{2}+a_{3} t^{3}+a_{4} t^{4}+a_{5} t^{5}+a_{6} t^{6}+a_{7} t^{7}, \\
& L_{\text {Sine }}=a_{0} \sin \left(a_{1} t+a_{2}\right)+a_{3} \sin \left(a_{4} t+a_{5}\right), \\
& L_{\text {Rational }}=\frac{a_{0}}{\left(t^{5}+a_{5} t^{4}+a_{4} t^{3}+a_{3} t^{2}+a_{2} t+a_{1}\right)}, \ldots \ldots \\
& L_{\text {Gausssian }}=a_{0} \exp \left(-\left(\left(t-a_{1}\right) / a_{2}\right)^{2}\right)+a_{3} \exp \left(-\left(\left(t-a_{4}\right) / a_{5}\right)^{2}\right),
\end{aligned}
$$

$$
L_{\text {Exponential }}=a_{0} \exp \left(a_{1} t\right)+a_{2} \exp \left(a_{3} t\right)
$$




$$
\begin{aligned}
L_{\text {Fourier }}= & a_{1}+a_{2} \cos \left(a_{0} t\right)+a_{3} \sin \left(a_{0} t\right)+a_{4} \cos \left(2 a_{0} t\right) \\
& +a_{5} \sin \left(2 a_{0} t\right),
\end{aligned}
$$

where $a$ is the unknown coefficient, $t$ is the time, $L$ is the inter-particle distance.

\section{Discussions}

\subsection{Simulation of Particle Movement with Capillary Force}

To evaluate the performance of the identical, nonidentical force schemes and curve fitting method, a series of data is extracted from a simple mathematical simulation, including the elapsed time, the inter-center distance, and the corresponding attractive force and velocity. This data will be used in sub-sections of 4.2 and 4.3. We assume two spherical particles with a radius of $20 \mu \mathrm{m}$ at a separation of $100 \mu \mathrm{m}$ trapped at the interface between liquid steel and inert gas. The attractive capillary force drives the two particles together to reduce the free energy of the system. ${ }^{7}$ ) The following physical properties of the molten steel (Fe20 wt.\% $\mathrm{Cr})$ and particles $\left(\mathrm{Ce}_{2} \mathrm{O}_{3}\right)$ are used: the densities of the molten steel and the particles are $6920 \mathrm{~kg} / \mathrm{m}^{3}\left(\rho_{\text {steel }}\right)$ and $6900 \mathrm{~kg} / \mathrm{m}^{3}\left(\rho_{\text {in }}\right),{ }^{23,24)}$ the surface tension of the molten steel is $1.58 \mathrm{~N} / \mathrm{m}(\gamma)$ at $1550^{\circ} \mathrm{C},{ }^{25)}$ and the contact angle between the steel and the particle is $105^{\circ}(\alpha) .{ }^{26)}$ According to the theory of capillarity, the asymptotic expressions for the capillary energy and capillary force between two spherical particles are presented by the Eqs. (16) and (17)

$$
\begin{gathered}
\Delta W_{\text {cap }}=2 \pi \gamma Q_{1} Q_{2} \ln \frac{\gamma_{\mathrm{e}} q L}{2}, \quad r_{\mathrm{c}, k}<<L<<q^{-1}, k=1,2 \ldots \\
F_{\text {cap }}=2 \pi \gamma \frac{Q_{1} Q_{2}}{L}, \quad r_{\mathrm{c}, k}<<L<<q^{-1}, k=1,2 \ldots \ldots .
\end{gathered}
$$

where $\Delta W_{\text {cap }}$ is the capillary interaction energy, $F_{\text {cap }}$ is the capillary force, $L$ denotes the inter-center distance, $\gamma_{\mathrm{e}}$ is a constant which is equal to $1.78107,{ }^{7)} r_{\mathrm{c}, k}$ is the radius of the circular three-phase contact line, $q^{-1}$ $\left(q=\sqrt{\left(\rho_{\text {steel }}-\rho_{\text {gas }}\right) g / \gamma}\right)$ is the capillary length, $Q_{k}$ is the socalled effective 'capillary charge' by analogy to Coulomb's law of electricity. The detailed procedure for calculating $Q_{k}$ can be found elsewhere. ${ }^{7,12)}$

At the inter-center distance of $100 \mu \mathrm{m}$, the capillary energy between two $20 \mu \mathrm{m}$ particles at $1550^{\circ} \mathrm{C}$ is around $39 k T$, where $k$ is the Boltzmann constant $(\mathrm{J} / \mathrm{K}), T$ is the temperature $(\mathrm{K})$. This strong interaction energy indicates that the capillary interaction is large enough to overcome the randomizing or disorganizing effect of the particles' thermal motion. By the driving of this attractive capillary force, the movement of the particles was simulated by running a MATLAB script with a time step of $0.033 \mathrm{~s}$. The data, including high precision inter-particle distances, the corresponding attractive force and velocity, were recorded during the simulation. The relationship of the inter-center distance vs time is shown in Fig. 5, and the capillary force at different time is demonstrated in the insert figure. One can see that the capillary force is non-linearly dependent on time.

\subsection{Evaluation of the Fitting Functions}

When doing the curve fitting, the coefficients of the curve functions are determined by the least square method, in which the sum of the squares of the residuals between the actual value and the predicted value is minimized. Since the capillary force is non-linearly dependent on the time, the lowest 4th order polynomial is applied. The coefficient for the polynomial function is shown in Table 1 with a coef-

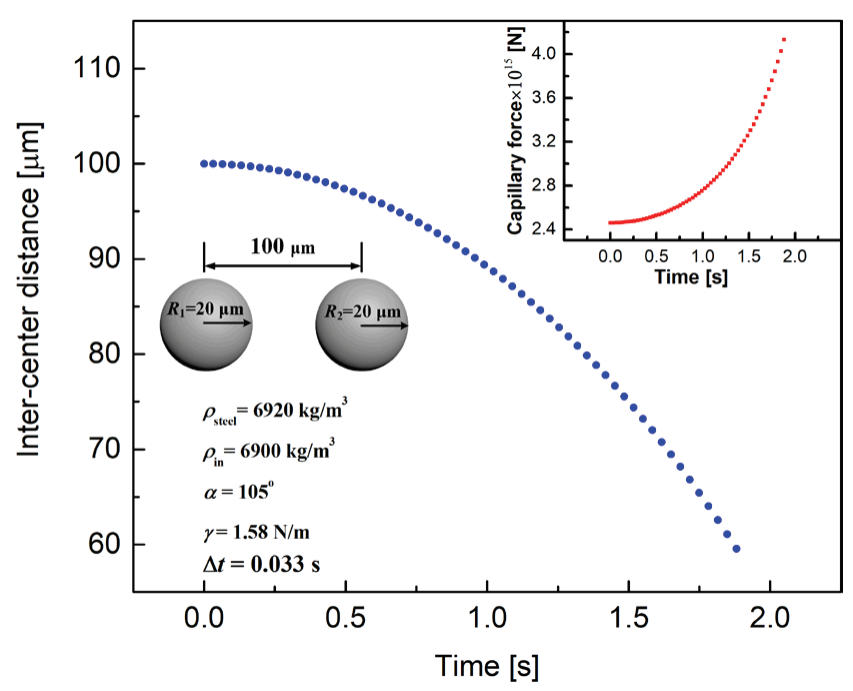

Fig. 5. The inter-center distance vs time as calculated for two spherical particles approaching each other with the driving of the capillary force. The initial separation distance is 100 $\mu \mathrm{m}$, the radius of the two particles is $20 \mu \mathrm{m}(R)$, and the time step is $0.033 \mathrm{~s}$. The insert shows the relationship between the capillary force and time. (Online version in color.)

Table 1. Coefficients and $R^{2}$ for different fitting functions: polynomial, sum of sines, rational, Gaussian function, exponential, and Fourier series. The highly accurate distance data from the simulation is used.

\begin{tabular}{ccccccccc}
\hline Functions & \multicolumn{9}{c}{ Coefficients } & \multicolumn{3}{c}{$R^{2}$} \\
\hline Poly-4th & $a_{0}$ & $a_{1}$ & $a_{2}$ & $a_{3}$ & $a_{4}$ & $a_{5}$ & Distance & Force \\
Sine-2nd & 99.992 & 0.126 & -11.07 & 0.533 & -0.4020 & - & 1 & 0.978 \\
Rational-5th & 164.410 & 0.719 & 1.420 & 65.02 & 0.985 & -1.846 & 1 & 0.902 \\
Gaussian-2nd & 2542.19 & 25.41 & 0.325 & 70.941 & 3.543 & -2.703 & 1 & -0.699 \\
Exponential-2nd & 94.396 & -0.171 & 2.010 & 26.988 & 1.621 & 1.188 & 1 & -1.20 \\
Fourier-2nd & 0.609 & -8.086 & 122.82 & 13.087 & -14.76 & -6.265 & 1 & 0.886 \\
\hline
\end{tabular}


ficient of determination $\left(R^{2}\right)$ of 1 . The value of $R^{2}$ indicates the goodness-of-fit, indicating a better fitting for values closer to unity. To keep the $R^{2}$ identical with the polynomial fitting, the lowest 2nd order of exponential, sine, Gaussian functions and Fourier series, and a 5th order rational function are adopted. The corresponding coefficients and $R^{2}$ are also listed in Table 1.

The velocities calculated by differentiating the aforementioned curve functions with respect to time are demonstrated in Fig. 6(a). The velocity derived from the inter-particle distance should be halved since the two identical particles are free to move. One can see that the predicted velocities from the curve functions agree well with the simulated velocity at most of the distances, with only a slight deviation at the two ends. The inertial force calculated from the fitting functions and from the simulation are shown in Fig. 6(b). One can see that significant deviations from the simulated force are detected both from the 2nd order Gaussian and the 5th order rational curves with a minus $R^{2}$. The predicted force from the exponential curve overestimates the inertial force at a short distance but underestimates it at a large distance. Among these fitting curves, the 4th order polynomial, the 2nd order sine and the 2nd Fourier predict the inertial force better at most of the distances. It is interesting to note that a good fitting in the distance can only guarantee a small residual between the predicted value and the real value at fitted data points; the slope of the curves between the data points can't be indicated but still can affect the predicted velocity and force significantly.

As introduced in section 2, a random error is usually introduced in the inter-particle distance measurement by the possible rotation of inclusion and the change of immersion depth. Apart from this random error, another error may arise if the distance is measured manually between two reference pixels. For instance, ideally, the centroid of a pixel is usually chosen as the reference point for the measurement. However, in practice, any position within the pixel could be chosen manually, introducing an error that can be equal to the dimension of the pixel. The dimension of an image pixel varies with the screen magnification during recording, as introduced in section 2. The general trend of the distance and time relationship could be captured by the data smoothing algorithms (e.g., the most used moving average) with proper parameters, but the force oscillation from the iteration schemes still exists. To evaluate the reliability of the fitting method, we will implement the curve fitting to the raw distance data without smoothing. To evaluate the effect of these errors, a noise term in the Gaussian distribution is added to each distance data point from the previous simulation. The mean of the Gaussian distribution is 0 , whereas the standard deviation (SD) varies with the magnitude of the error. For example, a large noise is added by assigning the SD with a value of the dimension of one image pixel represented by SDPx-1, indicating a less accurate distance data; or the noise term is much smaller where the SD equals to $10^{-6}$ times of the dimension of one image pixel with a short-hand notation of SDPx $-10^{-6}$, indicating a more accurate distance data.

In the previous analysis, the 4th order polynomial, the 2nd order sine and the 2nd order Fourier performed better than other functions on predicting the inertial force when the distance data is accurate. However, in practice, the accuracy of the measured distance is not always high. To imitate a low accurate distance, Gaussian noise with an SD of the dimension of a pixel $(0.7 \mu \mathrm{m}$, i.e., SDPx- 1$)$ is added to the simulated distance as shown in the insert in Fig. 7(a). The corresponding forces predicted from the fitting functions are also demonstrated in Fig. 7(a). The coefficients of these functions and the $R^{2} \mathrm{~s}$ for the fitted distance and the force are listed in Table 2. As can be seen, except for the exponential fitting, the $R^{2} \mathrm{~s}$ for the fitted distance are equal to 0.998 , which are smaller than the fittings without the noise term. The $R^{2} \mathrm{~s}$ for all the fitted forces also decrease compared to those without the noise term, in which the $R^{2}$ for the Fourier series decreases considerably from 0.886 to -3.47 . Although the $R^{2}$ for the 4 th order polynomial and the 2nd order sine functions decrease by the noise term, they still predict the inertial force better at most of the distances. It is interesting to note that a 'hook' tail showed up at the beginning of the movement both in the functions a)

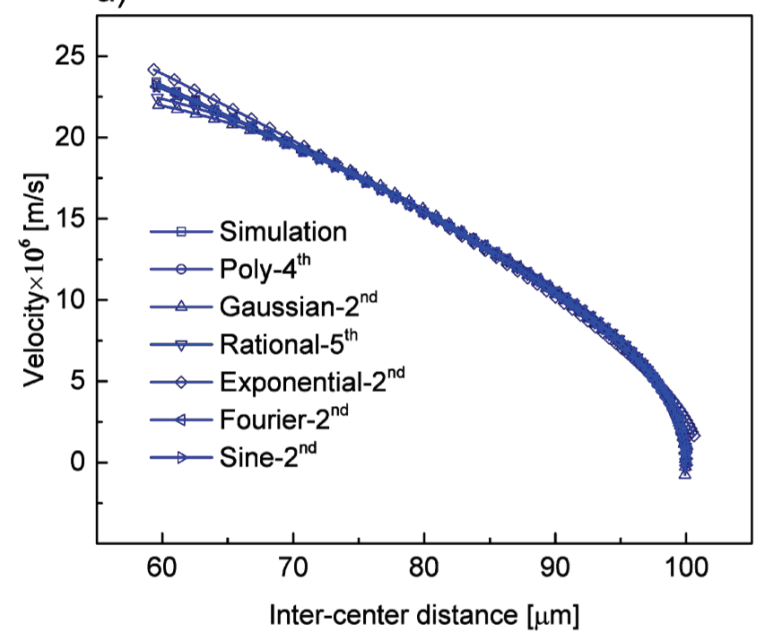

b)

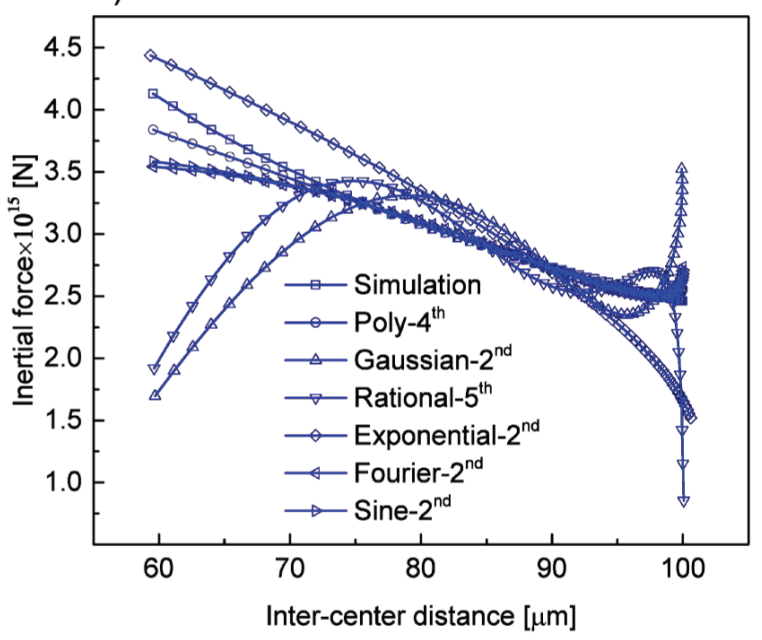

Fig. 6. The particle velocity and inertial force calculated from different distance-time fitting functions with the original high accurate distance date from the simulation: a) velocity vs inter-center distance, b) inertial force vs intercenter distance. (Online version in color.) 

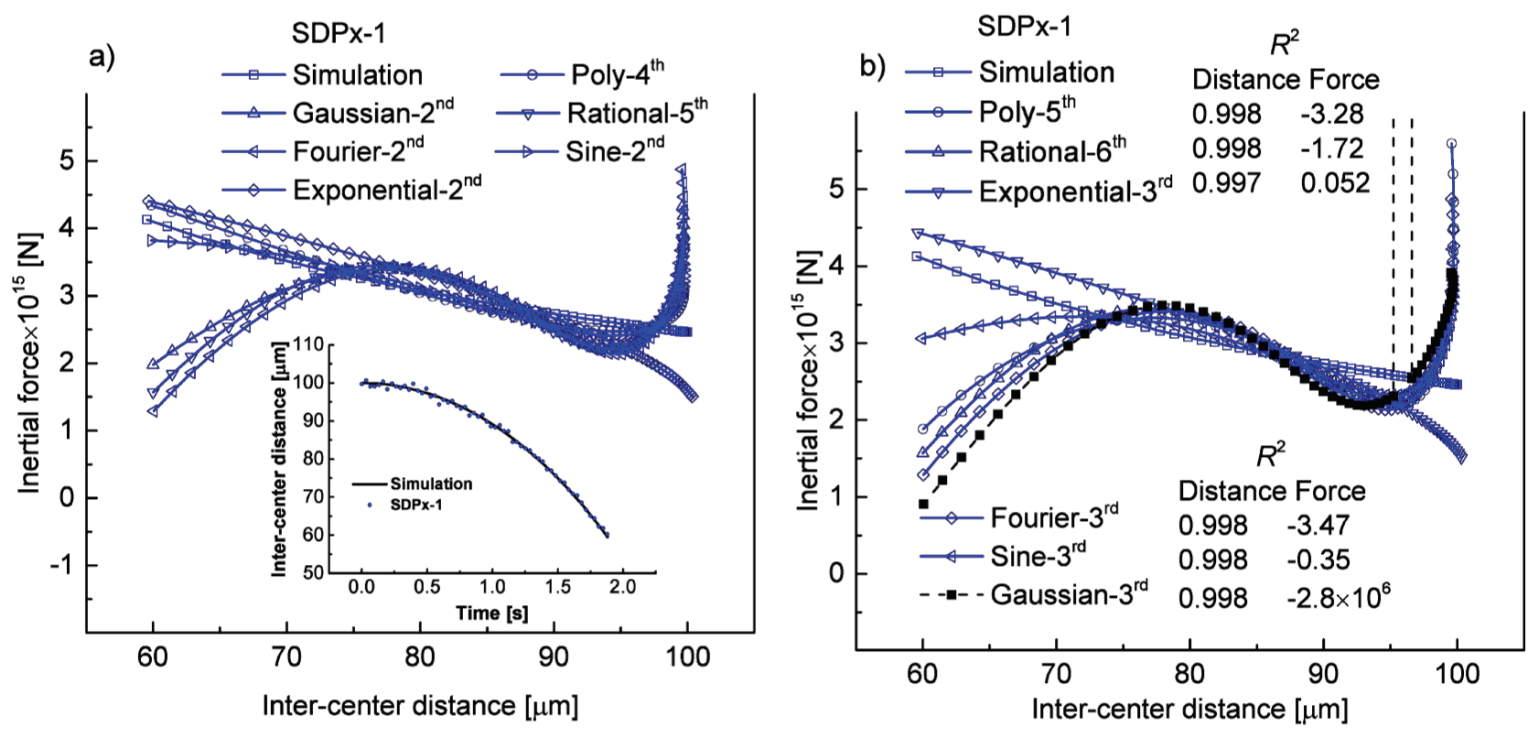

Fig. 7. Inertial force calculated from different orders of fitting functions with an additional noise of SDPx-1. The dimension of one image pixel is assumed to be $0.7 \mu \mathrm{m}$. a) the 4th order polynomial, the $2 \mathrm{nd}$ order of sine, exponential, Fourier series, and Gaussian functions, and the 5 th order rational function, insert is the corresponding distance vs time. b) the 5th order polynomial, the 3rd order of sine, exponential, Gaussian functions and Fourier series, and the 6th order rational function. (Online version in color.)

Table 2. Coefficients and $R^{2}$ for the fitting functions. The $\mathrm{SD}$ of the additional noise term equals to the dimension of one image pixel $(0.7 \mu \mathrm{m})$.

\begin{tabular}{ccccccccc}
\hline Functions & \multicolumn{9}{c}{ Coefficients } & \multicolumn{4}{c}{$R^{2}$} \\
\hline & $a_{0}$ & $a_{1}$ & $a_{2}$ & $a_{3}$ & $a_{4}$ & $a_{5}$ & Distance & Force \\
\hline Poly-4th & 99.732 & 1.142 & -13.561 & 2.716 & -0.968 & - & 0.998 & 0.769 \\
Sine-2nd & 104.556 & 0.622 & 1.404 & 6.336 & 1.714 & -2.573 & 0.998 & 0.549 \\
Rational-5th & 5055.84 & 50.744 & -1.278 & 8.532 & -1.723 & -0.507 & 0.998 & -1.72 \\
Gaussian-2nd & 92.7635 & -0.223 & 1.689 & 42.647 & 1.587 & 1.247 & 0.998 & -1.74 \\
Exponential-2nd & 94160.1 & 0.330 & -94059.8 & 0.330 & - & - & 0.997 & 0.064 \\
Fourier-2nd & 68.184 & 32.739 & 13.052 & -1.348 & -5.073 & 1.241 & 0.998 & -3.47 \\
\hline
\end{tabular}

of the 4th order polynomial and the 2nd order sine, where the predicted force drops down fast from $100 \mu \mathrm{m}$ and then increases gently afterward. The corresponding $R^{2} \mathrm{~s}$ of the fitted force are reduced by the appearance of this tail. One might imagine that increasing the order of these functions would improve their performance in predicting the inertial force. Figure 7(b) shows the inertial force derived from the fitting functions with one order higher. Contrary to our intuition, the higher-order fitting functions don't improve their performance in predicting the inertial force. Especially for the 3rd order Gaussian, a sharp jump appears at around $95 \mu \mathrm{m}$, results in an $R^{2}$ of $-2.8 \times 10^{6}$. We conclude that the 4 th order polynomial and 2 nd order sine are relatively robust fitting options to derive the inertial force from less accurate distance data.

\subsection{Comparison between the Identical, Non-identical Force Schemes and Curve Fittings}

In section 3, we discussed different methods to calculate the inertial force on moving particles, including the identical and non-identical force schemes and the curve fittings. To evaluate the performance of these methods, the inertial forces calculated from discrete distance data points with two degrees of noise, SDPx-1 and SDPx- $10^{-6}$, are shown in Fig. 8. Here, in the iteration schemes, the two particles are free to move. Figure 8(a) demonstrates that the iterated forces both from the identical and non-identical force schemes oscillate significantly around zero when the inter-particle distance is less accurate (SDPx-1). Here, the positive and negative forces represent an attractive and a repulsive force, respectively. It is worth noting that the capillary driving force on the particles is always positive in the simulation. Thus, we call the negative force due to the iteration schemes a 'spurious' repulsive force. In the research from Jiang et al. ${ }^{19)}$ an oscillating force is derived from the identical force scheme. However, the calculated negative force may not be a real but a 'spurious' repulsive force. One can see that the force oscillation from the non-identical force scheme is much stronger than from the identical force scheme, and the amplitude of the oscillation can be three orders larger than the simulated force. If the amplitude of the force is adopted to represent the inertial force, this approximation will overestimate the real force significantly. The force oscillation disappears from the iteration schemes when the noise decreases to SDPx- $10^{-6}$, see Fig. 8(b). Only the relative robust 4 th order polynomial and 

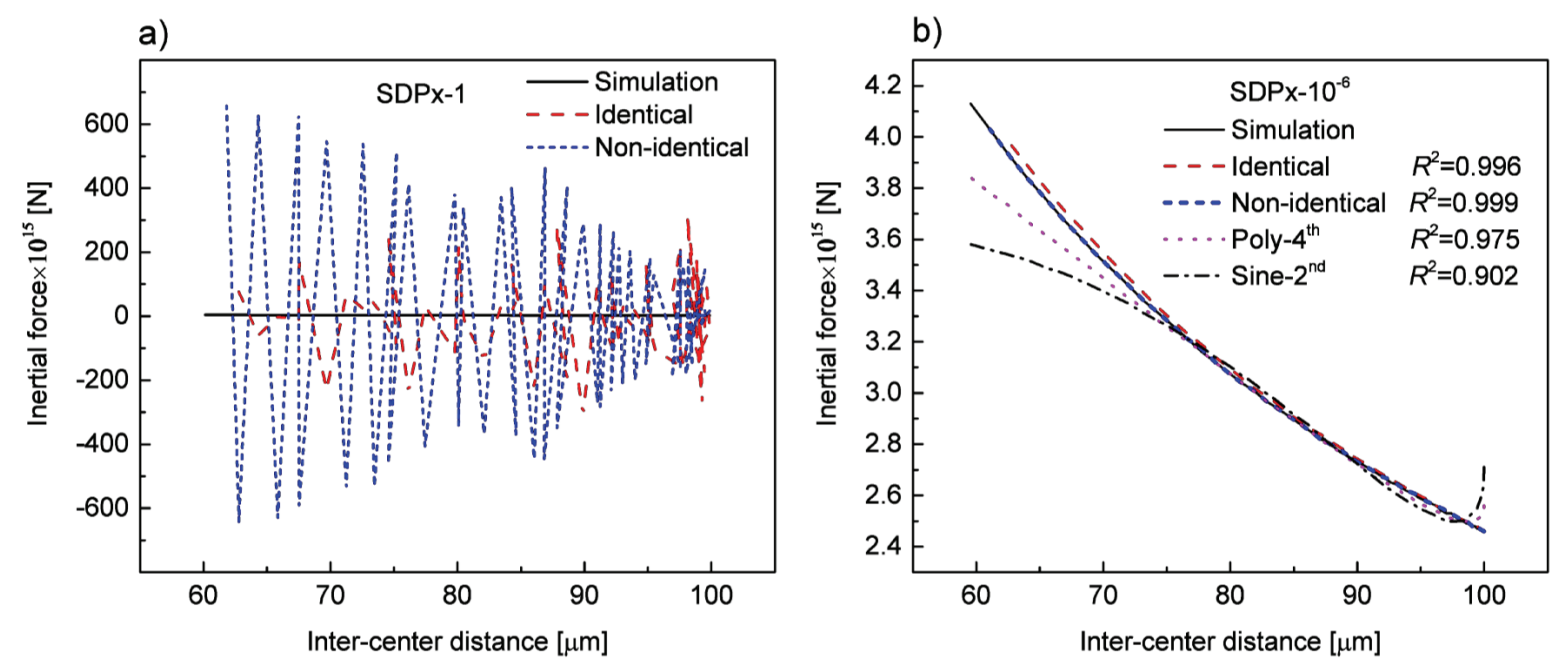

Fig. 8. a) inertial force calculated from identical and non-identical force schemes with a noise of SDPx-1, b) inertial force calculated from identical and non-identical force schemes, and fittings of 4th order polynomial and 2nd order sine with a noise of SDPx- $10^{-6}$. The dimension of one image pixel is assumed to be $0.7 \mu \mathrm{m}$. (Online version in color.)

2nd sine functions are used to do the fitting. Comparing the $R^{2}$ s from different methods, the iteration schemes perform better than the curve fittings in predicting the inertial force. Especially for the non-identical force scheme, an almost identical force is predicted to the real force at all studied distances. It is interesting to note that the 'hook' tail still appears in fittings of the 4th order polynomial and the 2 nd order sine with highly accurate distance data.

\subsection{Inertial Force Calculation from CSLM Image Sequence}

As a case study, the clustering behavior of $\mathrm{Ce}_{2} \mathrm{O}_{3}$ dominant inclusions at the interface of a $\mathrm{Fe}-20 \mathrm{wt} \% \mathrm{Cr}$ stainless steel melt and argon gas at $1550^{\circ} \mathrm{C}$ was observed in-situ in a CSLM furnace (type 1LM21H-SVF17SP). The steel sample was prepared in a resistive vertical tube furnace (GERO HTRV 100-250/18 with MoSiO2 heating elements). $45.0 \mathrm{~g}$ of electrolytic iron (Sigma-Aldrich $99.8 \%$ ) and 12.10 g chromium (Alfa Aesar 99.99\%) were melted in a high purity magnesia crucible (29 mm ID, $35 \mathrm{~mm}$ OD and $50 \mathrm{~mm}$ height) at $1600^{\circ} \mathrm{C}$ under the protection of purified argon, followed by adding $3.25 \mathrm{~g}$ of Fe $3.25 \% \mathrm{Ce}$ alloy. The liquid steel was sucked out by a quartz tube ( $5 \mathrm{~mm}$ ID) 5 min after the alloy addition and quenched in water immediately. The steel rod sample was sliced into discs and mirror polished for the CSLM test. The inclusions from the steel matrix were detected to be $\mathrm{Ce}_{2} \mathrm{O}_{3}$ dominant with minor impurities of $\mathrm{Si}$ and $\mathrm{S}$ through SEM-EDS analysis (Philips XL 30 FEG). For the CSLM observation, the mirror-polished steel sample was firstly heated up to $1500^{\circ} \mathrm{C}$ in an alumina crucible $(4.5 \mathrm{~mm}$ ID and $5.5 \mathrm{~mm} \mathrm{OD)} \mathrm{with} \mathrm{a} \mathrm{rate} \mathrm{of} 200^{\circ} \mathrm{C} / \mathrm{min}$, then $5^{\circ} \mathrm{C} /$ min to the observation temperature $\left(1550^{\circ} \mathrm{C}\right)$. The images were recorded 30 frames/second with a screen magnification of $700(0.7 \mu \mathrm{m} / \mathrm{pixel})$ after the liquid steel surface became quiescent. The physical properties of the molten steel and the inclusions, and the wetting properties are identical as used in the simulation in section 4.1. The time elapse of the relative positions between two pairs of inclusions, denoted by case 1 and case 2, are shown in Fig. 9. The areas of the inclusion pairs are 532.5 and $207.9 \mu \mathrm{m}^{2}$ in case $1,732.5$

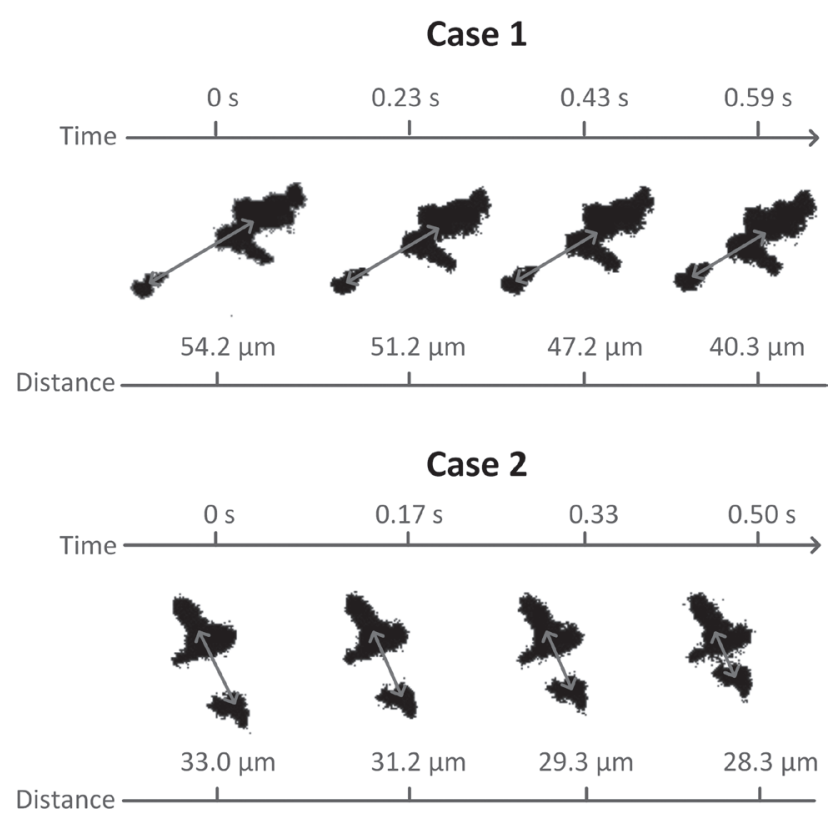

Fig. 9. Sequence of two pairs of inclusions attracting each other. The two pairs of inclusion are denoted by case 1 and case 2 , respectively.

and $142.3 \mu \mathrm{m}^{2}$ in case 2 , respectively. Note that the inclusions are assumed to be plates with a uniform thickness of $2 \mu \mathrm{m} .{ }^{8,11)}$ The specific inter-center distance is measured with the procedure proposed in section 2 .

Considering the stability and accuracy of the curve functions in predicting the inertial force, the 4th order polynomial and the 2nd order sine were used to fit the discrete distance points, as shown in Fig. 10. The specific coefficients and the $R^{2}$ s of the two curve functions are listed in Table 3. The polynomial curve and the sine curve are equally good to fit the distance points with an identical $R^{2}$ in both cases. One can see that the experimental points are distributed randomly at both sides of the fitted curves, which is similar to the insert figure in Fig. 7(a) with less accurate distance data.

In most inclusion clustering studies with the observation 
by CSLM, the inertial force is calculated from the identical force scheme. ${ }^{6,10,14,19)}$ Here, the inertial forces derived from the identical and non-identical force iteration schemes and the curve-fitting method are compared, see Fig. 11. The

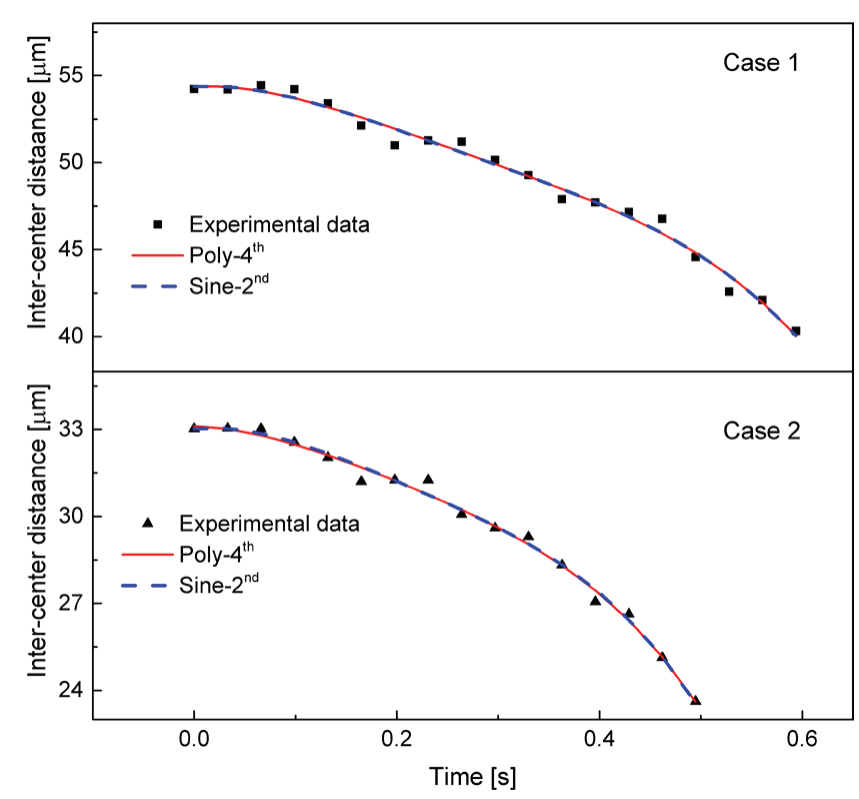

Fig. 10. The inter-center distance vs time for case 1 and case 2 . The discrete distance points are fitted by the 4 th order polynomial and the 2 nd order sine functions. (Online version in color.)

Table 3. The coefficients and $R^{2}$ for the two cases of the 4 th order polynomial and the 2 nd order sine curves.

\begin{tabular}{cccccccc}
\hline Functions & $a_{0}$ & $a_{1}$ & $a_{2}$ & $a_{3}$ & $a_{4}$ & $a_{5}$ & $R^{2}$ \\
\hline Poly-4th-case1 & 54.39 & 2.906 & -126.3 & 306.5 & -287.0 & - & 0.988 \\
Sine-2nd-case1 & 159.53 & 3.244 & 0.840 & 108.27 & 3.779 & 3.779 & 0.988 \\
Poly-4th-case2 & 33.11 & -0.6051 & -76.41 & 214.4 & -274.6 & - & 0.993 \\
Sine-2nd-case2 & 2.141 & 4.358 & 0.767 & 2.109 & 4.390 & 3.902 & 0.993 \\
\hline
\end{tabular}

a)

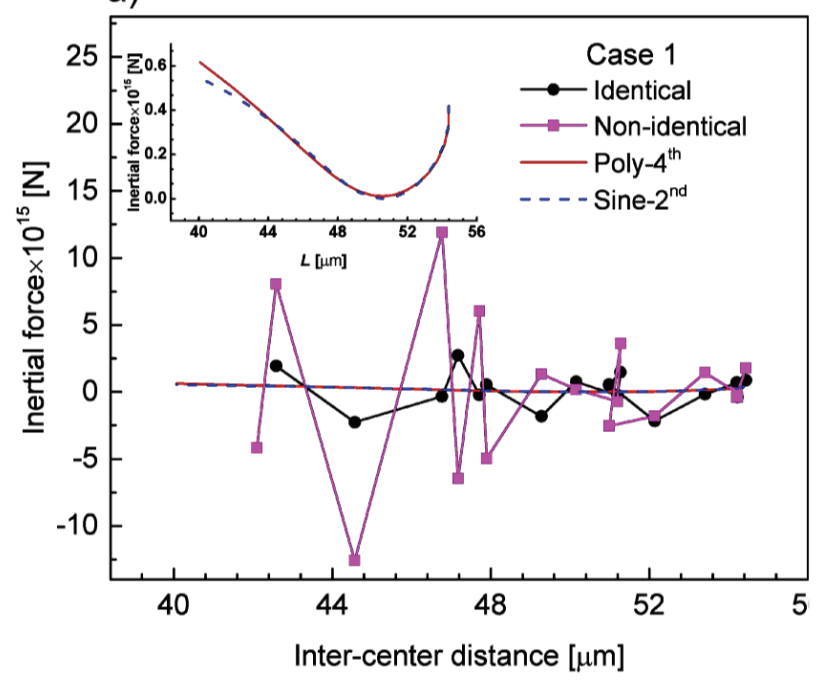

insert figures are the fitted forces by the 4th order polynomial and the 2 nd order sine, but with a smaller scale in the force axis. In both cases, the predicted force from the iteration schemes oscillates significantly around 0 , in which the force oscillation from the non-identical force scheme is more prominent than from the identical force scheme. The amplitude of the oscillation from the former and the latter schemes can be 2 and 1 orders higher than the fitted force in both cases. Thus, when a less accurate inter-particle distance is measured, it is hard to derive a valid inertial force from the iteration schemes. Note that one and two force points are dropped at a short distance from the non-identical and identical force schemes, respectively, due to the nature of the schemes.

As can be seen, both the 4th order polynomial and the 2 nd order sine curves show a similar trend in predicting the inertial force. The 'hook' tail appears in both cases at the end of the curve. According to the theory of capillarity, the attractive capillary force is inversely dependent on the interparticle distance, and the particle velocity increases when they approach each other. As a result, the distance vs time curve tends to be flat at the beginning of the movement as demonstrated in Fig. 10, where the slope of the fitting curves is more sensitive to the degree of the measurement errors. This sensitivity at the long distance may be the reason for the appearance of the 'hook' tails. As the capillary theory indicates, the inertial force is expected to monotonously decrease when the inter-center distance increases. ${ }^{7)}$ Thus, we approximately take the valid inertial force into account from the shortest distance to the bottom of the 'hook' tail, where the minimum forces are $6.6 \times 10^{-18} \mathrm{~N}$ in case 1 and of $4.4 \times 10^{-17} \mathrm{~N}$ in case 2 . Within the range from the short distance to the bottom of the 'hook' tail, the calculated inertial force shows a similar behavior as the inertial force indicated in Figs. 7(a) and 8(b). As a result, some of the force data at the long distance should be dropped, but a reasonable trend of the force is captured at a relatively short distance compared to the iteration schemes.

Fig. 11. The inertial force calculated from the experimental image sequence with the methods of identical and nonidentical force schemes, curve fittings of the 4th order polynomial and the 2 nd order sine. The inserts are the fitted forces but with a smaller scale in the force axis. a) case 1, b) case 2. (Online version in color.) 


\section{Conclusion}

To study the clustering behavior of inclusions at the interface of molten steel and inert gas, it is essential to calculate the long-range attractive force between particles accurately. We compared the inertial force calculated from the simulated distance data and CSLM image sequence by using different iteration schemes and curve-fitting functions. The main remarks are

(1) Both the inter-surface and inter-center distance can be used to represent the separation distance between inclusions. Using the inter-center distance is more likely to obtain an accurate inter-particle distance.

(2) A new non-identical force scheme is proposed to calculate the inertial force on particles from the discrete inter-particle distance points. It predicts the inertial force excellently only when the measured distance is accurate enough $\left(\mathrm{SDPx}-10^{-6}\right)$. The conventional identical force scheme also acquires high accurate inter-particle distance data to avoid unreasonable force oscillation.

(3) For the commonly used fitting functions, e.g., polynomial, exponential, sum of sines, rational, Gaussian functions and Fourier series, they all performed well to fit the discrete distance points. However, the inertial force derived from these functions differs from each other significantly. Among these functions, the 4th order polynomial and the 2nd order sine show the most robust prediction of the inertial force, even when the measured inter-particle distance is less accurate.

(4) Theoretically, the non-identical force scheme should be ideal for predicting the inertial force on moving particles. However, a considerable deviation from the real force is obtained when the inter-particle distance measured is less accurate. To ensure a valid and non-oscillatory inertial force, the measure distance should have an error less than $10^{-7} \mu \mathrm{m}$, but this high-accuracy requirement is almost impossible to be achieved by the current techniques with CSLM. The curve fitting method seems a better alternative to derive the inertial force, but it should be very careful when dealing with the second derivative of the fitting functions. The 4 th order polynomial and the 2 nd order sine are proper functions in predicting the inertial force on particles, while a 'hook' tail usually appears at a long distance that would lose some force data.

\section{Acknowledgment}

The authors thank the China Scholarship Council (CSC) for financial support (File No. 201706080018).

\section{REFERENCES}

1) A. L. V. da Costa e Silva: J. Mater. Res. Technol., 7 (2018), 283. https://doi.org/10.1016/j.jmrt.2018.04.003

2) V. Seshadri, C. A. da Silva, I. A. da Silva and E. da Silva Araújo Júnior: Tecnol. Metal. Mater. Min., 9 (2012), 22. https://doi.org/ 10.4322/tmm.2012.004

3) E. A. Chichkarev: Metallurgist, 54 (2010), 236. https://doi.org/ 10.1007/s11015-010-9286-1

4) H. Bai and B. G. Thomas: Metall. Mater. Trans. B, 32 (2001), 707. https://doi.org/10.1007/s11663-001-0125-4

5) L. Zheng, A. Malfliet, P. Wollants, B. Blanpain and M. Guo: ISIJ Int., 56 (2016), 926. https://doi.org/10.2355/isijinternational. ISIJINT-2015-561

6) H. Yin, H. Shibata, T. Emi and M. Suzuki: ISIJ Int., 37 (1997), 936. https://doi.org/10.2355/isijinternational.37.936

7) V. N. Paunov, P. A. Kralchevsky, N. D. Denkov and K. Nagayama: J. Colloid Interface Sci., 157 (1993), 100. https://doi.org/10.1006/ jcis. 1993.1163

8) H. Shibata, H. Yin and T. Emi: Philos. Trans. R. Soc. A, 356 (1998), 957. https://doi.org/10.1098/rsta.1998.0200

9) H. Yin, H. Shibata, T. Emi and M. Suzuki: ISIJ Int., 37 (1997), 946. https://doi.org/10.2355/isijinternational.37.946

10) W. Mu, N. Dogan and K. S. Coley: Metall. Mater. Trans. B, 48 (2017), 2379. https://doi.org/10.1007/s11663-017-1027-4

11) S. Kimura, K. Nakajima and S. Mizoguchi: Metall. Mater. Trans. B, 32 (2001), 79. https://doi.org/10.1007/s11663-001-0010-1

12) K. Nakajima and S. Mizoguchi: Metall. Mater. Trans. B, 32 (2001), 629. https://doi.org/10.1007/s11663-001-0118-3

13) S. Kimura, Y. Nabeshima, K. Nakajima and S. Mizoguchi: Metall. Mater. Trans. B, 31 (2000), 1013. https://doi.org/10.1007/s11663000-0077-0

14) B. Coletti, B. Blanpain, S. Vantilt and S. Sridhar: Metall. Mater. Trans. B, 34 (2003), 533. https://doi.org/10.1007/s11663-003-0021-1

15) Y. Kang, B. Sahebkar, P. R. Scheller, K. Morita and D. Sichen: Metall. Mater. Trans. B, 42 (2011), 522. https://doi.org/10.1007/ s11663-011-9497-2

16) S. Vantilt, B. Coletti, B. Blanpain, J. Fransaer, P. Wollants and S. Sridhar: ISIJ Int., 44 (2004), 1. https://doi.org/10.2355/ isijinternational.44.1

17) P. A. Kralchevsky, V. N. Paunov, N. D. Denkov, I. B. Ivanov and K. Nagayama: J. Colloid Interface Sci., 155 (1993), 420. https://doi.org/ 10.1006/jcis.1993.1056

18) P. A. Kralchevsky, V. N. Paunov, I. B. Ivanov and K. Nagayama: J. Colloid Interface Sci., 151 (1992), 79. https://doi.org/ 10.1016/0021-9797(92)90239-I

19) M. Jiang, X. Wang, J. Pak and P. Yuan: Metall. Mater. Trans. B, 45 (2014), 1656. https://doi.org/10.1007/s11663-014-0095-y

20) J. Wikström, K. Nakajima, H. Shibata, A. Tilliander and P. Jönsson: Ironmaking Steelmaking, 35 (2008), 589. https://doi.org/ $10.1179 / 174328108 \times 284589$

21) J. Appelberg, K. Nakajima, H. Shibata, A. Tilliander and P. Jönsson: Mater. Sci. Eng. A, 495 (2008), 330. https://doi.org/10.1016/ j.msea.2007.12.051

22) N. Otsu: IEEE Trans. Syst. Man Cybern., 9 (1979), 62. https://doi.org/ 10.1109/TSMC.1979.4310076

$23)$ W. Mu, N. Dogan and K. S. Coley: Metall. Mater. Trans. B, 48 (2017), 2092. https://doi.org/10.1007/s11663-017-0998-5

24) A. Sharan, T. Nagasaka and A. W. Cramb: Metall. Mater. Trans. B, 25 (1994), 939. https://doi.org/10.1007/BF02662777

25) K. Mori, M. Kishimoto, T. Shimose and Y. Kawai: J. Jpn. Inst. Met., 39 (1975), 1301. https://doi.org/10.2320/jinstmet1952.39.12 1301

26) B. J. Keene: Slag Atlas, ed. by Verein Deutscher Eisenhuttenleute (VDEh), Verlag Stahleisen GmbH, Düsseldorf, (1995), 530. 